\title{
Why the left ventricle is not a sphere
}

\author{
Felix A Blyakhman, Tatyana F Shklyar, Ivan A Pavlov, Sergey Yu Sokolov, Alexey A Grinko \\ Physics Department, Ural State University, Ekaterinburg, Russia
}

\begin{abstract}
In this study, we have tried to understand why the left ventricle (LV) is not a homogeneous sphere. An experimental model of a spherical ventricle was developed. The chamber was configured as a mathematical model, and the wall properties were represented by isolated cardiac muscles. The stroke work of the spherical LV when modelling different types of inhomogeneity in the wall structure was investigated. It was found that the emergence of even slight inhomogeneity in a spherical ventricle inevitably results in a diminution of pump function. It was concluded that at a given level of the myocardial contractility, a homogeneous spherical LV would not have any functional reserve, ie no ability to maintain pump function in case of additional load. Functional reserve can be achieved only with a certain degree of inhomogeneity. Thus, inhomogeneity in the normal left ventricular wall structure constitutes a strategic functional reserve that is absent in a homogeneous spherical ventricle.
\end{abstract}

Keywords: left ventricle, myocardium, pump function, contractility, inhomogeneity, functional reserve

\section{Introduction}

The normal left ventricle (LV) is a structurally inhomogeneous pump according to factors such as its geometry (Lessick et al 1996), the intricacy of the muscular fibre architecture (Streeter et al 1969, 1973), and the peculiarities of the myocardial blood supply and conduction systems (Scher 1953; Arisi et al 1983). Left ventricular structural complexity results in a spatial and temporal inhomogeneity of the chamber wall function (Hotta 1967; Lew and LeWinter 1986; Rademakers et al 1994; Takayama et al 2002). This phenomenon manifests as a difference in regional stresses, shortening, thickening and lengthening (Greenbaum and Gibson 1981; Villarreal and Lew 1990; Balzer et al 1999; Delepine et al 1999; Krahwinkel et al 2000).

Thus, inhomogeneity is known to be an indispensable attribute of the normal LV (Barletta et al 1998; Bogaert and Rademakers 2001); however, the significance of inhomogeneity for the normal heart has not yet been clarified. What is the reason for creating such an inhomogeneous device? It is implicitly assumed that inhomogeneity provides for a certain optimisation of pump efficiency and/or muscle function (Lew and LeWinter 1986; Brutsaert 1987; Hexeberg et al 1991; Blyakhman 1999; Rijcken et al 1999). In physical terms, however, it is difficult to imagine that the performance of an inhomogeneous muscular pump could be higher than that of a homogeneous one such as a sphere.

In the present study, we have sought an answer to the question: why isn't the LV a homogeneous muscular sphere?
To answer the question, a hybrid model of the ventricular chamber was developed. Muscle properties are represented by two strips of ventricular muscle effectively in series, and chamber properties are represented by a mathematical model. With this hybrid experimental/mathematical model, different degrees of inhomogeneity could be simulated and the impact of inhomogeneity on stroke work investigated.

The experimental data lead to the conclusion that at a given level of myocardial contractility, a homogeneous spherical LV would not have any functional reserve, ie no ability to maintain pump function in the case of additional load. It was found that functional reserve could be achieved only with a certain degree of inhomogeneity in LV wall structure. Hence, inhomogeneity is present in a normal ventricle to create functional reserve.

\section{Methods}

To simulate to some extent the inhomogeneity in a spherical LV wall we developed an experimental model. This model is based on a two-muscle interaction principle proposed many years ago (Tyberg et al 1969). A servo system for the registration and control of the mechanical activity of two isolated cardiac muscles was created. The system components are outlined in Figure 1.

The muscles were connected serially by means of the computer feedback control. To do this, the muscles' force

Correspondence: Felix Blyakhman, Physics Department, Ural State University, 5 I Lenin Ave, Ekaterinburg 620083, Russia; tel +73432

756 548; fax: +73432616778; email felix.bljakhman@usu.ru 


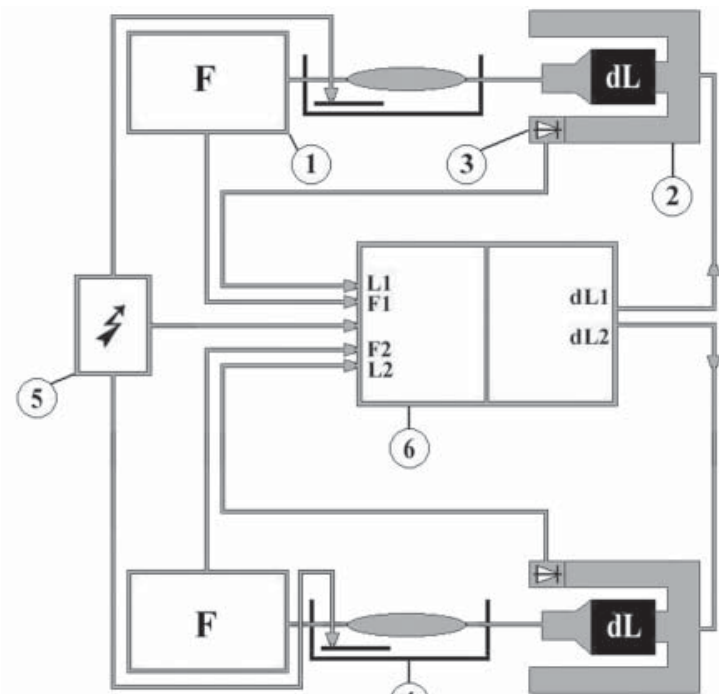

(4)

Figure I Schematic diagram of the experimental apparatus for the ventricular mechanics modelling. The system contains two independent devices, each including a force-transducer (I), a linear motor for applying mechanical deformations (2), an optical transducer to measure the muscles' lengths (3), and a sustenance system (4) with the bath for muscle perfusion and platinum electrodes connected to the electronic generator (5) (Blyakhman 1999). The measuring and control devices are interfaced with a computer (6).

had to be maintained equal, and this was done by redistribution of the muscles' lengths dynamically by means of servomotors. The computer feedback control included a geometrical model of the LV in the form of a sphere, whose wall properties were represented by two muscles. Two assumptions for a spherical LV model were used: (1) the thickness of the sphere's shell is far less than the sphere's radius; and (2) the sphere does not change shape during contraction.

The force produced by the cardiac muscles was converted into pressure by the Laplace equation:

$$
P=k_{f} F / R
$$

where $F$ is muscle force, $R$ is the radius of the model sphere and $k_{f}$ is the scale coefficient. The LV chamber radius and its change were calculated using the actual length of the muscles and their changes in length during the experiment:

$$
\begin{aligned}
& R=k_{l_{1}} l_{1}+k_{l_{2}} l_{2} \\
& \Delta R=k_{l_{1}} \Delta l_{1}+k_{l_{2}} \Delta l_{2}
\end{aligned}
$$

where $l_{1}$ and $l_{2}$ are the actual lengths of the muscles, and $k_{l_{1}}$ and $k_{l_{2}}$ are the coefficients for converting the actual lengths into the chamber radius. The chamber volume $(V)$ of the ventricle and the change in volume $(\Delta V)$ during a small time interval were calculated as:

$$
\begin{aligned}
& V=\frac{4}{3} \pi R^{3} \\
& \Delta V=4 \pi R^{2} \Delta R
\end{aligned}
$$

The variation of $k_{l_{1}}$ and $k_{l_{2}}$ coefficients enabled us to change the size of the sphere wall segments so that either one muscle dominated or neither muscle dominated. Thus, we had opportunity to simulate different extents of inhomogeneity.

Experiments $(\mathrm{n}=16)$ were performed on the cardiac muscles of frogs (Rana radibunda) that had been killed by decapitation. Two longitudinal samples from ventricle wall were dissected. Typically, samples were $3-5 \mathrm{~mm}$ in length and had a cross-section of about of $1-2 \mathrm{~mm}^{2}$. The Ringer solution of the following composition was used: $110 \mathrm{mmol} / \mathrm{L}$ $\mathrm{NaCl}, 2.5 \mathrm{mmol} / \mathrm{L} \mathrm{KCl}, 0.08 \mathrm{mmol} / \mathrm{L} \mathrm{KH}_{2} \mathrm{PO}_{4}, 2.4 \mathrm{mmol} / \mathrm{L}$ $\mathrm{Na}_{2} \mathrm{HPO}_{4}, 1.1 \mathrm{mmol} / \mathrm{L} \mathrm{CaCl}, 5.5 \mathrm{mmol} / \mathrm{L}$ glucose. The temperature of the bathing solution was $20-21^{\circ} \mathrm{C}$. The muscles were stimulated with rectangular pulses of $2-3 \mathrm{~ms}$

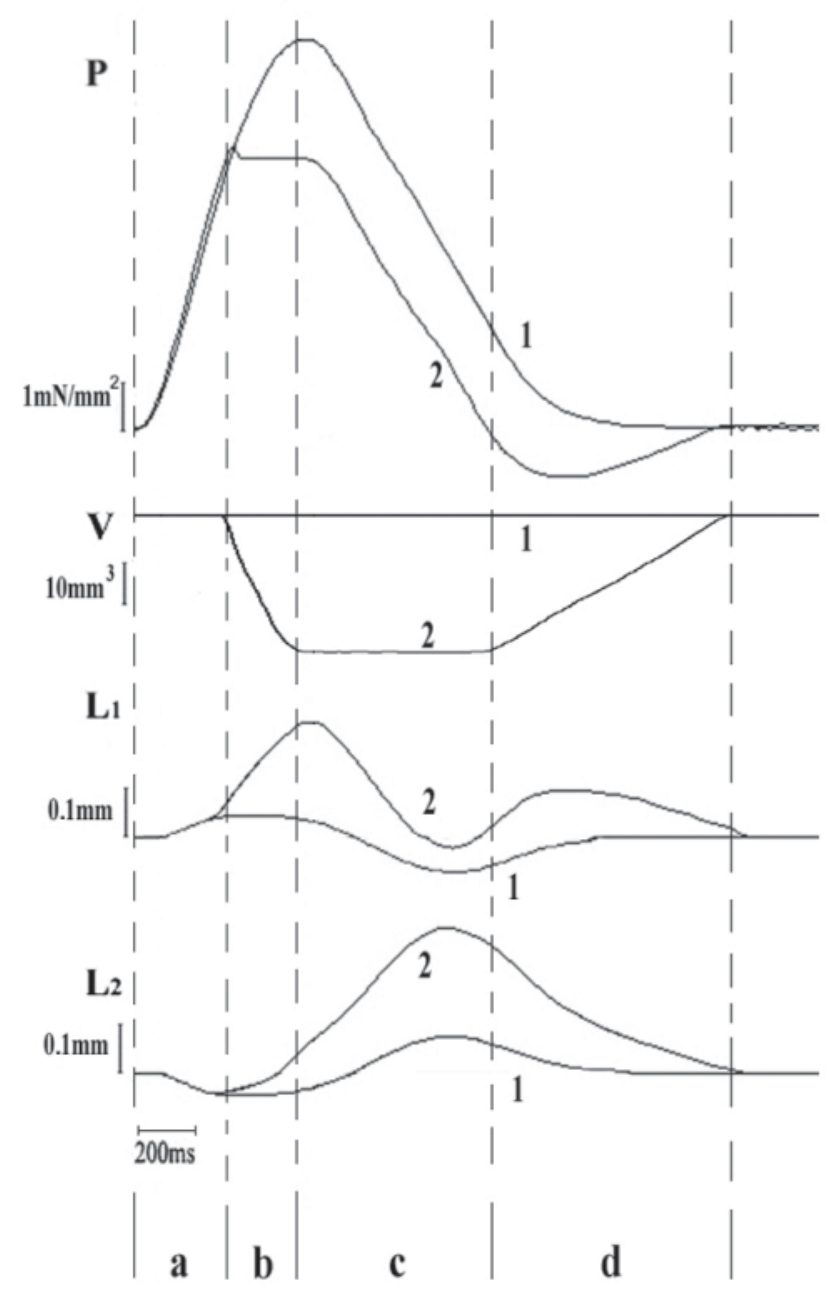

Figure 2 The experimental records example of the left ventricle (LV) mechanical parameters during the cardiac cycle. $P$ - pressure inside the spherical LV, obtained with the feedback control system; V - volume of the LV chamber, obtained with the feedback control system; $L_{1}, L_{2}-$ changes in length of the muscles (muscle shortening corresponds to the curve upward); I-trace for the isovolumic LV; 2 - trace obtained in course of the whole cardiac cycle modelling. Cardiac cycle phases: $\mathrm{a}$ - isovolumic contraction; $\mathrm{b}$ - ejection; $\mathrm{c}-$ isovolumic relaxation; $\mathrm{d}$ - diastolic filling. 
duration, applied by platinum electrodes. Stimulation rate was 20 beats/min.

Figure 2 demonstrates an example of the LV work modelling in course of the whole cardiac cycle. Two muscles with different cross-sections were chosen for examination. The coefficients $k_{l_{1}}$ and $k_{l_{2}}$ were set equal. The values of LV pre-load pressure and end-diastolic volume were specified initially. The traces labelled ' 1 ' correspond to LV isovolumic contraction when the post-load pressure is higher than the maximal possible pressure $\left(P_{0}\right)$ in the chamber at the given LV end-diastolic volume. In spite of the fact that LV volume is constant, a redistribution of the muscles' lengths takes place. The reason is a difference in the contractility between the two muscles. The traces labelled ' 2 ' correspond to the experimental condition when the LV post-load pressure was specified to be $\sim 70 \%$ of $P_{0}$. In this case, the feedback control realises the LV pump function in accordance with the main phases of the cardiac cycle. The contribution of each muscle to the ejection process is seen to be much different.

\section{Results}

Two different types of inhomogeneity in ventricular wall structure were simulated. Regional thickness difference in normal LV wall was modelled when two samples with different cross-sections were used. Temporal excitation difference between regions was simulated by using muscles with almost equal mechanical characteristics, but the delays between the electrical stimuli of the muscles were varied.

Figure 3 presents graphs of mechanical behaviour of muscles with initially different cross-sections, 'implanted' in the wall of the modelled spherical ventricle. In this case, at the same initial length, one muscle had an active force 1.5 times lower than the other. Traces labelled ' 1 ' correspond to conditions in which the weak wall segment area was five times smaller than the other one; this almost nullified the difference in LV regional thickness. Traces labelled '2' reflect the case when both segments had the same size. It means the maximal scale ratio between thick (strong) and thin (weak) segments. It is clearly seen that an inhomogeneity increase results in a decrease of ventricular stroke work, defined as the area inside the loop of the 'pressure-volume' curve.

The dependence of stroke work of the spherical LV on the extent of regional thickness inhomogeneity is shown in Figure 4. The LV wall segments as a percentage of the total area of modelled LV surface is plotted on the abscissa. LV stroke work is plotted on the ordinate. The stroke-work value $\mathbf{a}$
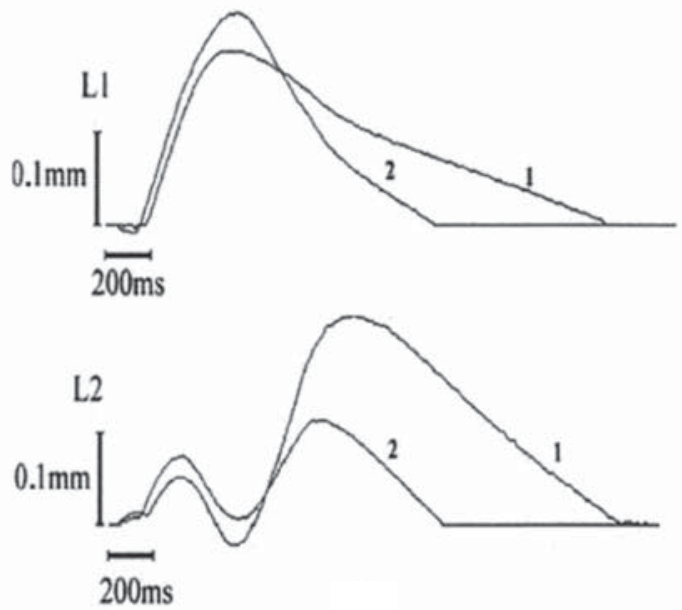

b

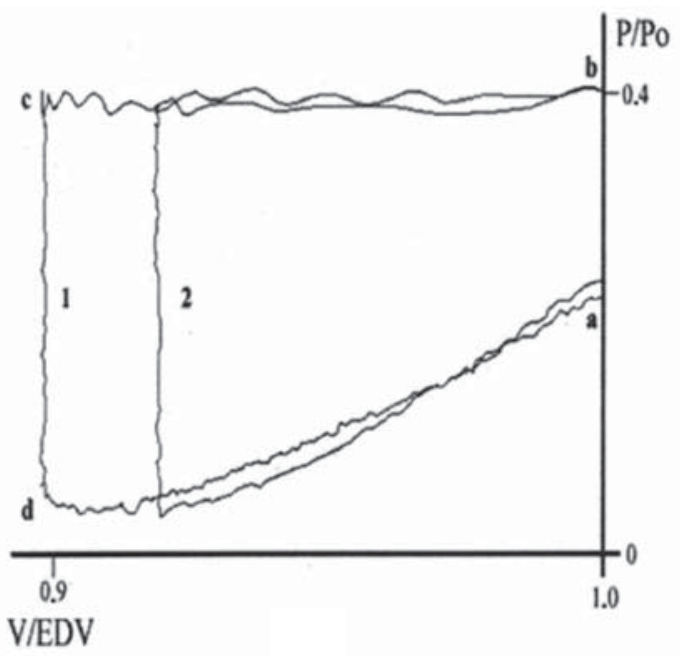

Figure 3 Mechanical behaviour of muscles 'implanted' in the spherical model left ventricle (LV) and their pump-function depending on the extent of regional thickness inhomogeneity. (a) The experimental records of the muscles' length changes over the course of the complete cardiac cycle modelling. $L_{1}$ - length changes of the thick sample; $L_{2}$ - length changes of the thin sample (muscle's shortening corresponds to the curve upward). (b) Pressure-volume loops obtained with the feedback control system. $x$-axis, LV volume as normalised to its end-diastolic value (EDV); $y$-axis, LV pressure as normalised to its maximal (isovolumic) value $\left(P_{0}\right)$. Cardiac cycle phases: a - isovolumic contraction; b ejection; $\mathrm{c}$ - isovolumic relaxation; $\mathrm{d}$ - diastolic filling. I, 2 - low and high extents of inhomogeneity, respectively (explanation in the text).

is normalised to that of a homogeneous sphere, ie when the model ventricle was represented by a single muscle only.

The data in Figure 4 show that the increase of scale inhomogeneity in LV wall results in a decrease of LV stroke work. So, the larger contribution of the thin region (weak muscle) to the total area of LV wall, the less the pump's efficacy.

The dependence of LV stroke work on the extent of temporal excitation difference between regions is shown in Figure 5. The data were obtained when both segments of modelled LV surface had the same size. The delays between electrical stimuli of muscles in percentage of the contraction 


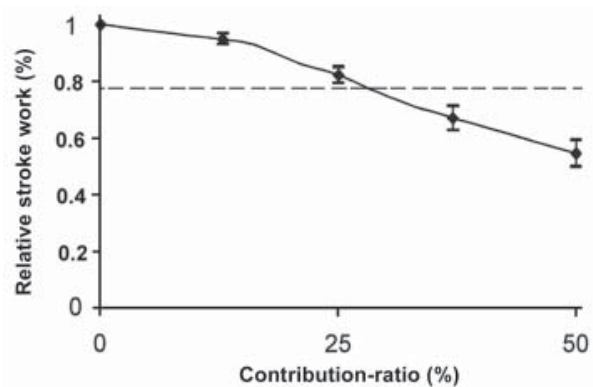

Figure 4 The dependence of spherical left ventricle (LV) stroke work on the extent of wall regional thickness difference.

cycle total duration are plotted on the abscissa. LV strokework values are plotted on the ordinate. The stroke work normalised to that of synchronous LV, ie the excitation delay was equal to zero.

According to the data (Figure 5), the temporal excitation difference between LV regions results in a decrease of the pump's stroke work. Similar to the data in Figure 4, the increase in inhomogeneity leads to the falling of LV efficacy.

Thus, independently of the sort of modelled inhomogeneity in the LV wall structure, an increase of inhomogeneity results in a decrease of spherical LV pump function.

\section{Discussion}

As is well known, an LV close in shape to a sphere is seen only in cases of severe myocardial damage. For example, the development of ischaemic heart disease (IHD) is accompanied by a progressive decrease of myocardial contractility, a differential decrease of regional wall thickness and an LV dilation nearing spherical shape (Kramer et al 1993; Swynghedauw 1999).

At the same time, notwithstanding impaired wall properties, the spherical LV ejects blood almost normally (Zardini et al 1993; Fantini et al 1994). Generally, transition of the LV from a non-spherical shape to a spherical one, myocardial remodelling, is of compensatory character and aims at maintaining adequate blood circulation (Cohn et al 2000). The spherically shaped pathological LV has minimal

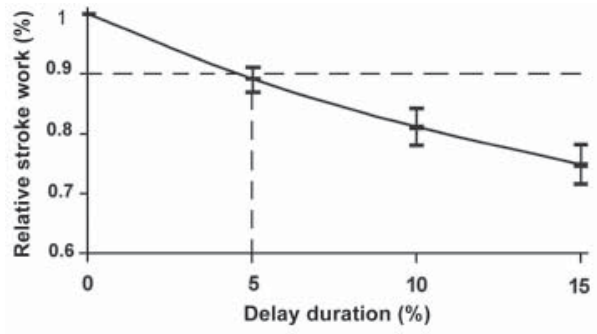

Figure 5 The dependence of stroke work of spherical left ventricle (LV) on the extent of temporal excitation difference between wall surface regions. functional reserve (Vokonas et al 1976; Wallis et al 1984; Sunnerhagen et al 1990; Krahwinkel et al 2000), ie it has no ability to maintain pump function in the case of additional load. Commonly, LV functional reserve is considered as the myocardium contractility reserve and is based on heart regulatory mechanisms such as the Frank-Starling law and effects of the heart rate or inotropic agents.

Thus, the development of cardiovascular pathology is accompanied by a decrease of LV functional reserve on the one hand, and by a progressive increase of myocardial remodelling on the other. In other words, as the myocardium contractility reserve is exhausted, the LV resorts to the help of structural transformations to keep LV pump function close to its normal value. However strange it seems, nature has apparently not made the normal LV a simple sphere, reserving it for extreme cases.

According to the data presented in Figures 4 and 5, the emergence of even slight inhomogeneity in a spherical ventricle inevitably results in a diminution of pump function. At first sight, this result does not fit with clinical observations, which confirm the pump's functional independence from the scale of myocardial inhomogeneity. This is caused by the absence of regulatory mechanisms to support functional reserve in the modelled pump, in contrast to the real heart.

As for experimental conditions, however, such reserve could come from the outside to maintain LV stroke-work stability. One can add, for example, a positive inotropic agent (say, adrenaline) to perfusion baths with muscles, to increase their contractile function. In that case, the higher the scale of inhomogeneity set, the larger the quantity of inotropic agent needed. In other words, we recruit myocardial contractility reserve to a greater extent. This interpretation reconciles the experimental data with clinical observations.

At a given level of myocardial contractility, a homogeneous spherical LV carries out maximal stroke work, ie it performs the maximal possible function and therefore has no functional reserve. When the work carried out is lower than maximal (eg the value marked with a dotted line in Figure 4 and Figure 5), the functional reserve emerges. It is necessarily accompanied by a certain degree of inhomogeneity in LV wall structure. For example, Figure 5 shows that for the supply of $10 \%$ LV functional reserve, $5 \%$ excitation delay is necessary. Thus, at a certain degree of myocardial contractility, the LV functional reserve is only possible in case of a certain degree of inhomogeneity in LV wall structure. 
As far as pump function is concerned, inhomogeneity diminishes LV performance relative to a homogeneous sphere but creates functional reserve. Therefore, initial inhomogeneity in the normal LV wall structure constitutes a strategic functional reserve. It is spent in extreme situations, be it an extra load or a pathological process.

Thus, inhomogeneity appears as an independent determinant of the LV functional reserve, which is maximal in the normal heart. Hence, LV is not a sphere because at a given level of myocardial contractility the functional reserve of a homogeneous pump is close to zero.

\section{Acknowledgements}

This investigation was supported by the Russian Foundation for Basic Research.

\section{References}

Arisi G, Macchi E, Baruffi S et al. 1983. Potential fields on the ventricular surface of the exposed dog heart during normal excitation. Circ Res, 52:706-15.

Balzer P, Furber A, Delepine S et al. 1999. Regional assessment of wall curvature and wall stress in left ventricle with magnetic resonance imaging. Am J Physiol, 277(3 Pt 2):H901-10.

Barletta G, Baroni M, Del Bene R et al. 1998. Regional and temporal nonuniformity of shape and wall movement in the normal left ventricle. Cardiology, 90:195-201.

Blyakhman FA. 1999. Do we know much about the heart? J Nat (Rus), 11:23-30.

Bogaert J, Rademakers F. 2001. Regional nonuniformity of normal adult human left ventricle. Am J Physiol Heart Circ Physiol, 280: H610-20.

Brutsaert DL. 1987. Nonuniformity: a physiologic modulator of contraction and relaxation of the normal heart. J Am Coll Cardiol, 9:341-8.

Cohn JN, Ferrari R, Sharpe N. 2000. Cardiac remodeling - concepts and clinical implications: a consensus paper from an international forum on cardiac remodeling. J Am Coll Cardiol, 35:569-82.

Delepine S, Furber A, Balzer P et al. 1999. MRI quantification of regional variations of left ventricular parietal stress in normal subjects [French]. Arch Mal Coeur Vaiss, 92:1189-96.

Fantini F, Barletta G, Di Donato M et al. 1994. Alterations in left ventricular shape in patients with angina and single-vessel coronary disease. Coron Artery Dis, 5:901-8.

Greenbaum RA, Gibson DG. 1981. Regional non-uniformity of left ventricular wall movement in man. Br Heart J, 45:29-34.
Hexeberg E, Matre K, Lekven J. 1991. Transmural fibre direction in the anterior wall of the feline left ventricle: theoretical considerations with regard to uniformity of contraction. Acta Physiol Scand, 141: 497-505.

Hotta S. 1967. The sequence of mechanical activation of the ventricle Jpn Circ J, 31:1568-72.

Krahwinkel W, Haltern G, Gulker H. 2000. Echocardiographic quantification of regional left ventricular wall motion with color kinesis. Am J Cardiol, 85:245-50.

Kramer CM, Lima JA, Reichek $\mathrm{N}$ et al. 1993. Regional differences in function within noninfarcted myocardium during left ventricular remodeling. Circulation, 88:1279-88.

Lessick J, Fisher Y, Beyar R et al. 1996. Regional three-dimensional geometry of the normal human left ventricle using cine computed tomography. Ann Biomed Eng, 24:583-94.

Lew WY, LeWinter MM. 1986. Regional comparison of midwall segment and area shortening in the canine left ventricle. Circ Res, 58:678-91.

Rademakers FE, Rogers WJ, Guier WH et al. 1994. Relation of regional cross-fiber shortening to wall thickening in the intact heart. Threedimensional strain analysis by NMR tagging. Circulation, 89 : 1174-82.

Rijcken J, Bovendeerd PH, Schoofs AJ et al. 1999. Optimization of cardiac fiber orientation for homogeneous fiber strain during ejection. Ann Biomed Eng, 27:289-97.

Scher AM, Young AC, Malmgren AL et al. 1953. Spread of electrical activity through the wall of the ventricle. Circ Res, 1:539-47.

Streeter DD, Hanna WT. 1973. Engineering mechanics for successive states in canine left ventricular myocardium. I. Cavity and wall geometry. Circ Res, 33:639-55.

Streeter DD, Spotnitz HM, Patel DP et al. 1969. Fiber orientation in the canine left ventricle during diastole and systole. Circ Res, 24 $339-47$.

Sunnerhagen KS, Bhargava V, Shabetai R. 1990. Regional left ventricular wall motion abnormalities in idiopathic dilated cardiomyopathy. Am J Cardiol, 65:364-70.

Swynghedauw B. 1999. Molecular mechanisms of myocardial remodeling. Physiol Rev, 79:215-62.

Takayama Y, Costa KD, Covell JW. 2002. Contribution of laminar myofiber architecture to load-dependent changes in mechanics of LV myocardium. Am J Physiol Heart Circ Physiol, 282:H1510-20.

Tyberg JV, Parmley WW, Sonnenblick EH. 1969. In-vitro studies of myocardial asynchrony and regional hypoxia. Circ Res, 25:569-78.

Villarreal FJ, Lew WY. 1990. Finite strains in anterior and posterior wall of canine left ventricle. Am J Physiol, 259(5 Pt 2):H1409-18.

Vokonas PS, Pizzada FA, Hood WD. 1976. Experimental myocardial infarction. Dynamical changes in segmental mechanical behavior of infarcted and non-infarcted myocardium. Am J Cardiol, 37:853-9.

Wallis DE, O'Connel JB, Henkin RE. 1984. Segmental wall motion abnormalities in dilated cardiomyopathy. $\mathrm{J} \mathrm{Am} \mathrm{Cell} \mathrm{Cardiol,} 4$ $674-82$

Zardini P, Marino P, Golia G et al. 1993. Ventricular remodeling and infarct expansion. Am J Cardiol, 72:98G-106G. 


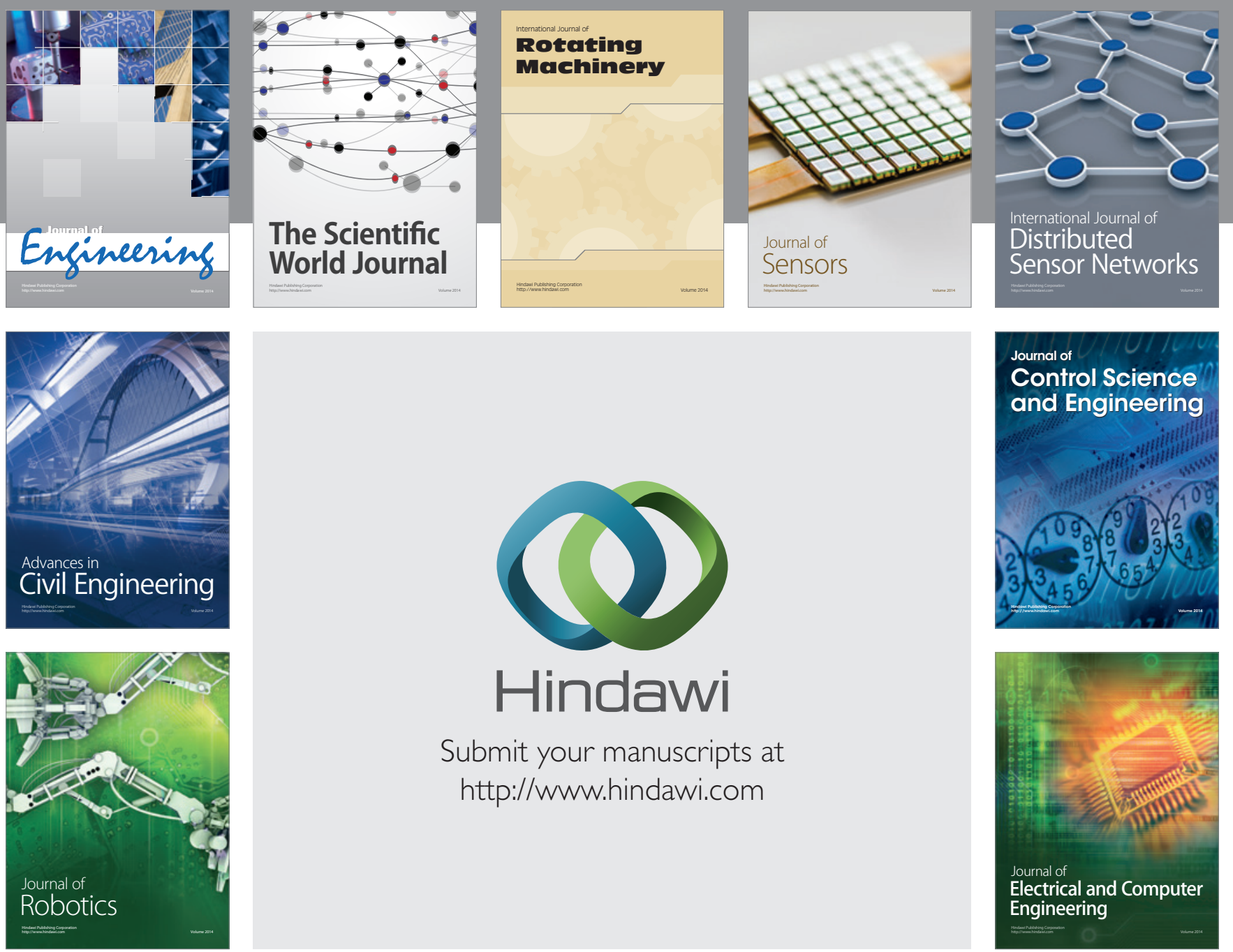

Submit your manuscripts at

http://www.hindawi.com
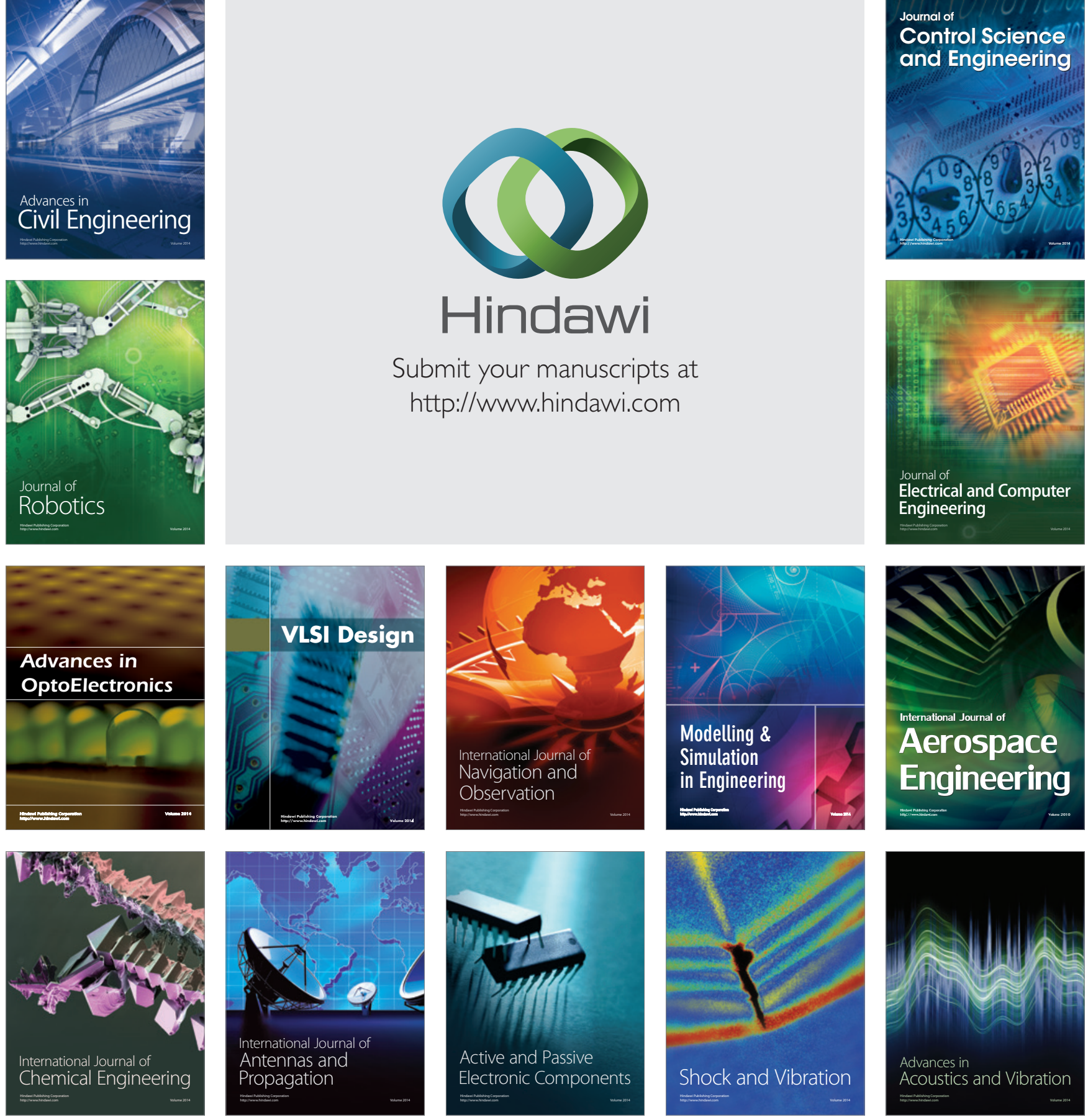